\title{
SOLVENT OPTIMIZATION in the RECRYSTALLIZATION PURIFICATION of PHARMACEUTICAL PRODUCTS
}

\author{
Richard Fickelscherer ${ }^{1}$ \\ ${ }^{1}$ Affiliation not available
}

August 31, 2020

\begin{abstract}
Abstract: This treatment describes the details of a methodical three step algorithm for determining the optimal operating conditions for the recrystallization separations of solid mixtures. Our algorithm was applied to optimally separate a representative pharmaceutical product (Caffeine) from a related pharmaceutical product (Theophylline). The limitations of such calculations with currently available, widely used predictive methods for computing solution thermodynamics without experimental data are directly examined. Also presented here is a novel two stage recrystallization procedure which can potentially dramatically improve the recovery yields of the desired products. The systematic optimization calculations described herein should enable researchers to quickly screen many potential solvent systems and operating conditions and concentrate experimental efforts only on the most promising candidates for such purifications.
\end{abstract}

\section{Hosted file}

SORPPP AIChE Paper V5.1 DS final paper.doc available at https://authorea.com/users/ 354915/articles/478378-solvent-optimization-in-the-recrystallization-purification-ofpharmaceutical-products 